\title{
ОЦЕНКА ВЛИЯНИЯ ТЕРМОБАРИЧЕСКИХ УСЛОВИЙ НА ЭФФЕКТИВНОСТЬ ПРОЦЕССА НИЗКОТЕМПЕРАТУРНОЙ АБСОРБЦИИ ПРИ ПРОМЫСЛОВОЙ ПОДГОТОВКЕ ГАЗА НА МЕСТОРОЖДЕНИИ КРАЙНЕГО СЕВЕРА
}

\author{
Кутуков Владислав Владимирович1, \\ Kutukov_Vlad@rambler.ru
}

\author{
Пономарёв Александр Иосифович1, \\ pnmrv@mail.ru
}

\author{
Чеботарёв Виктор Васильевич1, \\ kafedrargkm@mail.ru
1 Уфимский государственный нефтяной технический университет, Россия, 450062, г. Уфра, ул. Космонавтов, 1.

\begin{abstract}
Актуальность. Деэтанизированный газовый конденсат является ценным сырьем для нефртепереработки и нефртехимического производства, поэтому увеличение степени его извлечения из газа газоконденсатных месторождений на промысловых установках является актуальной научно-технической задачей.

Цель: обосновать возможность увеличения выхода нестабильного конденсата-фрракции С з+ из потока газа на действующей промысловой установке комплексной подготовки газа и конденсата одного из нефтегазоконденсатных месторождений Крайнего Севера за счет оптимизации рабочих параметров процесса низкотемпературной абсорбции.

объект: промысловая установка низкотемпературной абсорбции.

Meтод: моделирование процессов сепарации и низкотемпературной абсорбции в среде программного комплекса «PetroSim». Результаты. Исследовано влияние давления и температуры, расхода газа, удельного расхода и состава абсорбента - нестабильного конденсата, на эфффективность процесса подготовки газа и конденсата на компьютерной модели промысловой установки низкотемпературной абсорбции и низкотемпературной сепарации. Показано, что использование процесса низкотемпературной абсорбции на последней ступени сепарации для рассматриваемого состава сырого газа обеспечивает выход целевой фрракции $C_{3+}$ примерно в 2 раза больше в широком диапазоне давлений и температур по сравнению с процессом низкотемпературной сепарации. Обоснована возможность увеличения степени извлечения в товарный нестабильный конденсат фрракции $\mathrm{C}_{3+}$ на последней ступени сепарации в процессе низкотемпературной абсорбции на 21 \% только за счет оптимизации режимных параметров работы установки без изменения технологической схемы. При часовом расходе сырого газа 225 тыс. м3/4 оптимизацией термобарических параметров процесса низкотемпературной абсорбции - изменением давления с 3,75 до 5,0 МПа и температуры с минус 30 до минус $35^{\circ} \mathrm{C}$, извлечение фрракции $\mathrm{C}_{3+}$ в нестабильный товарный конденсат

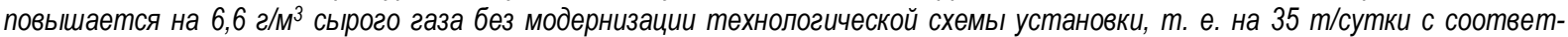
ствующим сокращением ее уноса с товарным газом. При «утяжелении» компонентного состава абсорбента путем снижения давления в разделителе нестабильного конденсата первой ступени сепарации степень извлечения фрракции $\mathrm{C}_{3+}$ в товарный нестабильный конденсат в низкотемпературном абсорбере повышается на $25 \%$, m. е. еще на 6,6 2/M³ , или 7 m/сутки дополнительно с соответствующим дальнейшим сокращением ее содержания в товарном газе. Но реализация такого режима уже требует минимальной модернизации установки низкотемпературной абсорбции путем врезки насоса в технологический трубопровод подачи нестабильного конденсата орошения с разделителя $P$-1 в низкотемпературный абсорбер, которая окупаема в короткие сроки.
\end{abstract}

\section{Ключевые слова:}

Газоконденсатное месторождение, промысловая подготовка газа, низкотемпературная абсорбция, компьютерное моделирование, термобарические условия, газ сепарации, выход нестабильного конденсата, абсорбент, насос.

\section{Введение}

Традиционной технологией извлечения нестабильного конденсата из газа на установках комплексной подготовки газа и конденсата (УКПГ), используемой газодобывающими компаниями на месторождениях Крайнего Севера, является низкотемпературная сепарация (НТС) на температурном уровне до минус $30{ }^{\circ} \mathrm{C}[1-5]$.

Данная технология была первой и практически остается единственной на газоконденсатных месторождениях России [6, 7]. Основным достоинством установок НТС является их относительная простота в них нет массообменных аппаратов и используется небольшое количество технологических аппаратов и отсутствуют массообменные процессы. К недостат- кам технологии НТС можно отнести невысокую степень извлечения нестабильного конденсата из газа с небольшим содержанием тяжёлых углеводородов [8]. На сегодняшний день большинство базовых месторождений ПАО «Газпром» находится на стадии падающей добычи со снижающимся конденсатным фактором. Установки низкотемпературной абсорбции (НТА) технологически несколько сложнее и более капиталоемки, но позволяют существенно сократить унос фракции $\mathrm{C}_{3+}$ с газом сепарации и соответственно увеличить выход нестабильного конденсата [9]. Однако и они периодически нуждаются в «подстройке» технологических режимов для обеспечения возможно полного извлечения нестабильного конденсата (НК) [10-12]. 
Основным отличием технологии НТА является использование низкотемпературных абсорберов на конечной ступени выделения конденсата из потока газа вместо низкотемпературных сепараторов $[13,14]$. Температурный режим работы низкотемпературного абсорбера обеспечивается за счет дроссель-эффекта, величина которого зависит от давления, перепада давления на дросселе и состава газа, подаваемого в аппарат. Чрезмерно глубокое снижение температуры конечной ступени сепарации нецелесообразно из-за высокой температуры помутнения нестабильного конденсата и образования парафиноотложений, а также значительного увеличения содержания метана и этана в нестабильном конденсате [13].

Повышение эффективности эксплуатации установки промысловой НТА может быть обеспечено в той или иной мере корректировкой термобарических параметров работы установки, совершенствованием массообменных секций абсорберов и/или применением селективного абсорбента [15-21]. Так, в работах $[16,17]$ предложена новая технология низкотемпературной абсорбции применительно к установке НТА рассматриваемого нефтегазоконденсатного месторождения для углубленного извлечения фракции $\mathrm{C}_{3+} \mathrm{c}$ использованием селективного абсорбента постоянного состава, получаемого и регенерируемого на специальной установке из нестабильного конденсата первой ступени сепарации, что в промысловых условиях технологически крайне сложно осуществить из-за изменяющегося состава скважинной продукции.

Поэтому после модернизации массообменных секций абсорберов заменой сетчатых тарелок на ре- гулярные насадки на первый план выходит задача определения области оптимальных значений технологических параметров процесса НТА при подготовке пластовой смеси текущего состава объекта разработки по технологической схеме, представленной на рис. 1. Задача несёт в себе ряд неопределенностей: состав нестабильного конденсата действующим регламентом УКПГ не установлен. Кроме того, конечной товарной продукцией добывающего комплекса в целом является газ (смесь газов сепарации и деэтанизации), подаваемый в магистральный газопровод, и деэтанизированный конденсат, направляемый в конденсатопровод протяженностью несколько сот километров на перерабатывающий завод.

\section{Методика проведения исследования}

Режимными параметрами установки НТА являются: давление, температура, расходы и составы входных потоков газа и нестабильного конденсата орошения, поступающих в низкотемпературный абсорбер. Под деэтанизированнным газовым конденсатом понимается жидкая фаза, состоящая при рабочих термобарических условиях процесса из углеводородов (УВ) группы $\mathrm{C}_{3+}$. На промысловых установках подготовки газа конденсат такого качества получать не удается, а из пластового газа получают нестабильный конденсат, состоящий как из УВ группы $\mathrm{C}_{3+}$, так и из более легких УВ - $\mathrm{C}_{1,2}$. Поэтому показателями эффективности работы низкотемпературного абсорбера являются: удельный выход нестабильного конденсата, извлечение фракции $\mathrm{C}_{3+}$ в нестабильный конденсат и содержание легких углеводородов $\left(\mathrm{C}_{1,2}\right)$ в НК.

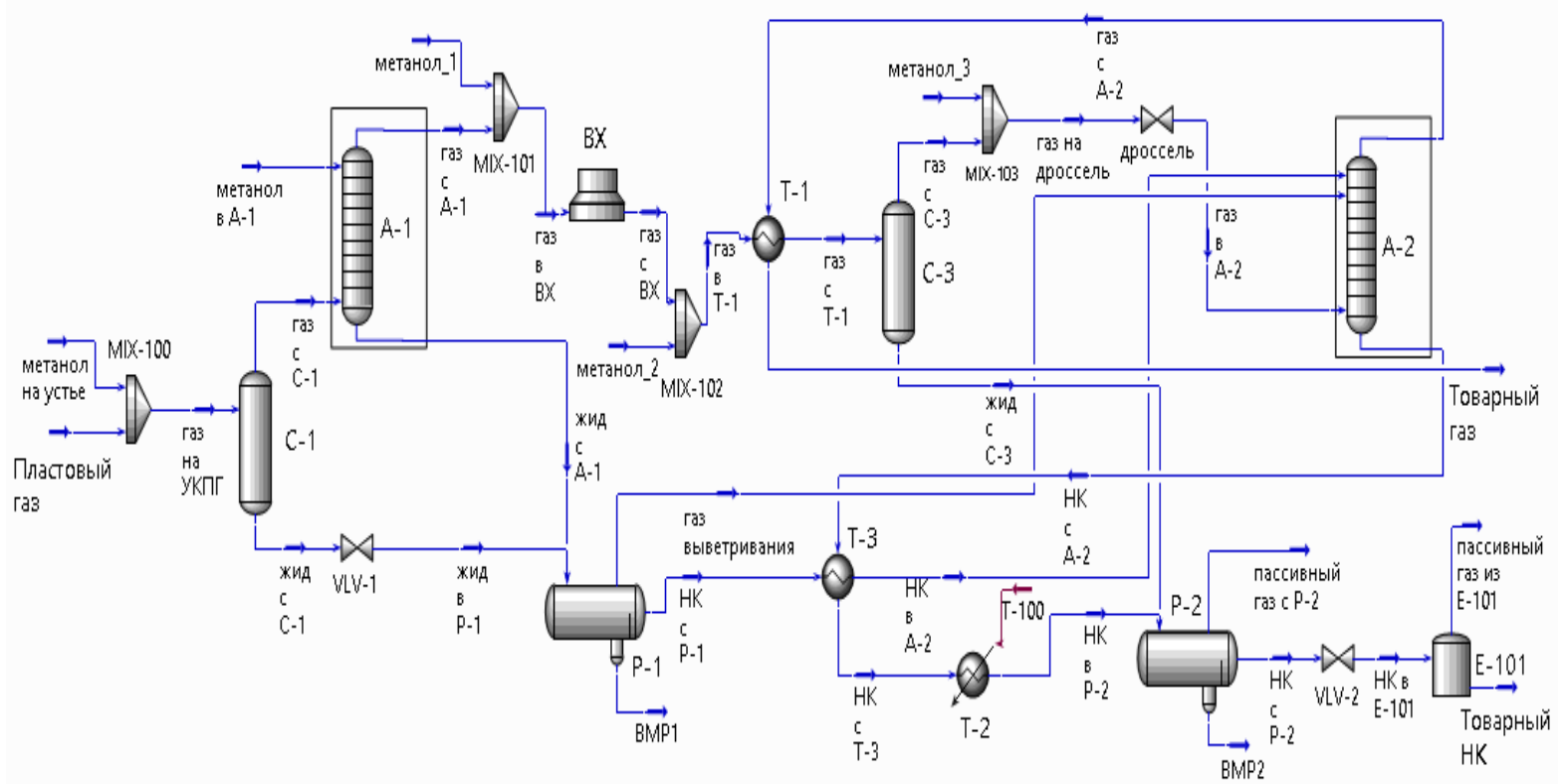

Pис. 1. Модель технологической схемы подготовки сырого газа с процессом НTA в программе «РеtroSim»: C-1, C-3 сепаратор; P-1, P-2 - трёхфазный разделитель; ВX-воздушный холодильник; T-1, T-2, T-3 - теплообменник; A-1 - абсорбер; A-2 - низкотемпературный абсорбер; MIX-100, 101, 102, 103 - место ввода метанола; VLV100, 102 - регулирующий клапан

Fig. 1. Model of the technological scheme of raw gas treatment by low-temperature absorption (LTA) in the program «PetroSim»: C-1, C-3 - separator; P-1, P-2 - 3-phase separator; BX-air cooler; T-1, T-2, T-3 - heat exchanger; A-1 absorber; A-2 - low-temperature absorber; MIX-100, 101, 102, 103 - methanol injection place; VLV-100, 102 - control valve 
Нахождение области оптимальных значений режимных параметров работы установки HТА осуществлялось путем анализа влияния давления, температуры и расходов входных потоков в установку на целевые показатели - выход НК и фракции $\mathrm{C}_{3+}$ $[22,23]$. Исследование проводилось с помощью программного комплекса технологического моделирования «PetroSim» компании KBC (Yokogawa), в котором была построена модель технологической нитки подготовки газа рассматриваемой установки с использованием модуля процесса НТА (рис. 1).

Симулятор позволяет проводить вычисления составов и теплофизических свойств равновесных фаз различных смесей в цепочке технологических процессов системы «пласт - скважина - коллекторустановка промысловой подготовки углеводородного сырья». Для получения достоверных прогнозных результатов расчета производилась предварительная адаптация модели объекта под имеющиеся промысловые данные в рабочем диапазоне параметров процесса.

На симуляторе расчет всей технологической нитки производился для составов сырого газа, поступающего на обработку в установку, и газа после первой ступени сепарации, направляемого на последнюю ступень сепарации через промежуточную C-3 (рис. 1) в низкотемпературный абсорбер (табл. 1).

Таблица 1. Компонентный состав пластового газа поступающего на установку комплексной подготовки газа и конденсата (УКПГ), и га за, направляемого в низкотемпературный абсорбер А-2

Table 1. Component composition of the formation gas supplied to the complex gas and condensate treatment plant (CGCTP), and gas entering the low-temperature absorber A-2

\begin{tabular}{|c|c|c|}
\hline \multirow[t]{2}{*}{$\begin{array}{l}\text { Компонент } \\
\text { Component }\end{array}$} & $\begin{array}{l}\text { Пластовый } \\
\text { газ } \\
\text { Formation } \\
\text { gas }\end{array}$ & $\begin{array}{c}\text { Газ, поступа- } \\
\text { ющий в A-2 } \\
\text { Gas entering in } \\
\text { the A-2 }\end{array}$ \\
\hline & \multicolumn{2}{|c|}{ \% мольные $/ \%$ molar } \\
\hline $\mathrm{C}_{1}$ & 90,66 & 91,5453 \\
\hline $\mathrm{C}_{2}$ & 4,71 & 4,5673 \\
\hline $\mathrm{C}_{3}$ & 1,83 & 1,6318 \\
\hline $\mathrm{i}-\mathrm{C}_{4}$ & 0,34 & 0,2690 \\
\hline$n-C_{4}$ & 0,44 & 0,3188 \\
\hline $\mathrm{i}-\mathrm{C}_{5}$ & 0,20 & 0,1063 \\
\hline $\mathrm{n}-\mathrm{C}_{5}$ & 0,14 & 0,0658 \\
\hline $\mathrm{C}_{6}$ & 0,28 & 0,0608 \\
\hline $\mathrm{C}_{7}$ & 0,29 & 0,0207 \\
\hline $\mathrm{C}_{8}$ & 0,27 & 0,0049 \\
\hline $\mathrm{C}_{9}$ & 0,10 & 0,0004 \\
\hline $\mathrm{C}_{10+}$ & 0,21 & 0,0002 \\
\hline $\mathrm{N}_{2}$ & 0,15 & 0,1527 \\
\hline $\mathrm{CO}_{2}$ & 0,38 & 0,3603 \\
\hline Метанол/Methanol & $-{ }_{-}$ & 0,7029 \\
\hline $\begin{array}{c}\text { Содержание } \mathrm{C}_{5+} \text { В газе, } \mathrm{\Gamma} / \mathrm{M}^{3} \\
\mathrm{C}_{5+} \text { content in gas, } \mathrm{g} / \mathrm{m}^{3}\end{array}$ & 63,7 & 14,71 \\
\hline $\begin{array}{c}\text { Содержание } \mathrm{C}_{3+} \text { в газе, } \mathrm{r} / \mathrm{M}^{3} \\
\mathrm{C}_{3+} \text { content in gas, } \mathrm{g} / \mathrm{m}^{3}\end{array}$ & 123,75 & 68,27 \\
\hline
\end{tabular}

Также для характеристики материального баланса процесса НТА в табл. 1, 2 представлены компонентные составы газа, поступающего в низкотемператур- ный абсорбер А-2 из абсорбера А-1, и нестабильного конденсата, направляемого на орошение в абсорбер A-2 с первой ступени сепарации, в зависимости от режима работы разделителя Р-1 в диапазоне давлений 2,0...6,0 МПа при температуре $20^{\circ} \mathrm{C}$.

Таблица 2. Компонентный состав абсорбента - нестабильного конденсата, поступающего на орошение в абсорбер A-2, в зависимости от режима работы трёхфазного разделителя первой ступени $P-1$

Table 2. Component composition of the absorbent unstable condensate entering the absorber A-2 for irrigation, depending on the operating mode of the three-phase separator $P-1$

\begin{tabular}{|c|c|c|c|c|c|c|c|c|c|}
\hline \multirow{3}{*}{$\begin{array}{c}\text { Компо- } \\
\text { нент } \\
\text { Compo- } \\
\text { nent } \\
\end{array}$} & \multicolumn{9}{|c|}{$\begin{array}{c}\text { НК на орошение A-2, \% массовые/мольные } \\
\text { UC for irrigation A- } 2, \% \text { mas/molar }\end{array}$} \\
\hline & \multicolumn{9}{|c|}{$\mathrm{P}(\mathrm{M} \Pi \mathrm{a}) /(\mathrm{MPa})$} \\
\hline & 2,0 & 2,5 & 3,0 & 3,5 & 4,0 & 4,5 & 5,0 & 5,5 & 6,0 \\
\hline \multirow{2}{*}{$\mathrm{C}_{1}$} & 1,37 & 1,81 & 2,27 & 2,76 & 3,27 & 3,80 & 4,36 & 4,94 & 5,54 \\
\hline & 8,05 & 10,34 & 12,64 & 14,94 & 17,23 & 19,52 & 21,78 & 24,03 & 26,26 \\
\hline \multirow{2}{*}{$\mathrm{C}_{2}$} & 1,34 & 1,51 & 1,66 & 1,78 & 1,89 & 1,99 & 2,07 & 2,15 & 2,23 \\
\hline & 21 & 4,62 & 4,93 & 5,15 & 5,32 & 5,44 & 5,53 & 5,59 & 5,63 \\
\hline \multirow{2}{*}{$\mathrm{C}_{3}$} & & 2,70 & 2,78 & 2,84 & 2,89 & 2,93 & 2,96 & 2,98 & 3,00 \\
\hline & 56 & 5,63 & 5,64 & 5,61 & 5,55 & 5,47 & 5,38 & 5,28 & 5,18 \\
\hline \multirow{2}{*}{$\mathrm{i}-\mathrm{C}_{4}$} & 1,25 & 1,27 & 1,28 & 1,29 & 1,29 & 1,29 & 1,29 & 1,29 & 1,29 \\
\hline & 2,04 & 2,01 & 1,97 & 1,93 & 1,88 & 1,83 & 1,78 & 1,73 & 1,69 \\
\hline \multirow{2}{*}{$\mathrm{n}-\mathrm{C}_{4}$} & 2,12 & 2,14 & 2,14 & 2,15 & 2,14 & 2,14 & 2,14 & 2,13 & 2,12 \\
\hline & 3,45 & 3,38 & 3,30 & 3,21 & 3,12 & 3,04 & 2,95 & 2,86 & 2,78 \\
\hline \multirow{2}{*}{$\mathrm{i}-\mathrm{C}_{5}$} & 2,34 & 2,33 & 2,32 & 2,31 & 2,30 & 2,28 & 2,27 & 2,26 & 2,24 \\
\hline & 3,07 & 2,97 & 2,88 & 2,78 & 2,70 & 2,61 & 2,52 & 2,44 & 2,36 \\
\hline \multirow{2}{*}{$\mathrm{n}-\mathrm{C}_{5}$} & 3 & 1,92 & 1,91 & 1,90 & 1,89 & 1,88 & 1,87 & 1,85 & 1,84 \\
\hline & 2,53 & 2,45 & 2,37 & 2,29 & 2,22 & 2,15 & 2,08 & 2,01 & 1,94 \\
\hline \multirow{2}{*}{$\mathrm{C}_{6}$} & 9,09 & 9,01 & 8,94 & 8,87 & 8,80 & 8,74 & 8,67 & 8,60 & 8,53 \\
\hline & 9,96 & 9,60 & 9,27 & 8,95 & 8,65 & 8,35 & 8,07 & 7,79 & 7,52 \\
\hline \multirow{2}{*}{ C- } & 15,82 & 15,68 & 15,55 & 15,43 & 15,31 & 15,18 & 15,06 & 14,94 & 14,81 \\
\hline & 1,92 & 14,37 & 13,87 & 13,39 & 12,93 & 12,49 & 12,06 & 11,64 & 11,23 \\
\hline \multirow{2}{*}{ C } & 21,13 & 20,94 & 20,76 & 20,59 & 20,43 & 20,26 & 20,10 & 19,93 & 19,76 \\
\hline & 7,47 & 16,83 & 16,24 & 15,67 & 15,13 & 14,61 & 14,11 & 13,62 & 13,14 \\
\hline \multirow{2}{*}{ c } & 9,92 & \begin{tabular}{|l|l}
9,83 \\
\end{tabular} & \begin{tabular}{|l|l|}
9,74 \\
\end{tabular} & 9,66 & \begin{tabular}{|l|l|}
9,59 \\
\end{tabular} & 9,51 & 9,43 & \begin{tabular}{|l|}
9,35 \\
\end{tabular} & \begin{tabular}{|l|l}
9,27 \\
\end{tabular} \\
\hline & 7,30 & 7,04 & 6,79 & 6,55 & 6,33 & 6,11 & 5,90 & 5,69 & 5,49 \\
\hline \multirow{2}{*}{$\mathrm{C}_{10+}$} & 29,65 & 29,38 & 29,13 & 28,90 & 28,66 & 28,43 & 28,20 & 27,96 & 27,72 \\
\hline & 5,79 & 16,17 & 15,60 & 15,06 & 14,54 & 14,04 & 13,56 & 13,09 & 12,63 \\
\hline \multirow{2}{*}{$\mathrm{CO}_{2}$} & 0,08 & 0,10 & 0,11 & 0,13 & 0,14 & 0,15 & 0,16 & \begin{tabular}{|l|l}
0,17 \\
\end{tabular} & 0,18 \\
\hline & 0,18 & 0,21 & 0,23 & 0,25 & 0,28 & 0,28 & 0,29 & 0,30 & 0,31 \\
\hline & 1,3 & 1,37 & 1,38 & 1,40 & 1,41 & 1,42 & 1,43 & 1,44 & 1,45 \\
\hline & 4,45 & 4,37 & 4,29 & 4,21 & 4,14 & 4,06 & 3,98 & 3,91 & 3,84 \\
\hline
\end{tabular}

Увеличение давления в разделительной емкости P-1 с 2,0 до 6,0 МПа приводит к значительному изменению углеводородного состава НК, подаваемого в абсорбер А-2: содержание компонентов $\mathrm{C}_{1}-\mathrm{C}_{2}$ в НК возрастает на 19,63\% мольн., или в 2,6 раза, содержание пропана $\left(\mathrm{C}_{3}\right)$ остается практически неизменным, а содержание фракций $\mathrm{C}_{4}-\mathrm{C}_{6}$ и $\mathrm{C}_{7+}$ снижается на 4,66 \% мольн., или в 1,3 раза, и на 13,99 \% мольн., или также в 1,3 раза, соответственно. Массовые концентрации углеводородных компонентов НК изменяются аналогично. Здесь отметим, что «облегчение» состава НК ведет к снижению его абсорбционной способности $[15,17]$.

Для анализа работы низкотемпературного абсорбера выбран диапазон давлений 3,0..6,0,0 МПа. Нижнее значение обусловлено минимальным давлением в межпромысловом коллекторе, а верхнее соответствует максимально возможному давлению в абсорбере А-2 при существующих технологических возможно- 
стях. Область изучения влияния температуры процесса ограничивается технологически допустимым минимальным ее значением по ряду причин: высокая температура помутнения нестабильного конденсата минус $36{ }^{\circ} \mathrm{C}$; значительное увеличение содержания в НК газа дегазации; ограничение по минимально допустимым рабочим температурам материала технологического оборудования (не ниже минус $36{ }^{\circ} \mathrm{C}$ ). Поэтому для исследования влияние температуры на процесс НТА была выбрана область минус 28... минус $35^{\circ} \mathrm{C}$.

Расход газа ограничен плановыми показателями по добыче газа и количеством рабочих технологических ниток и принят в расчетах постоянным и равным 225 тыс. ${ }^{3} /$ ч. Подача НК с трехфазного разделителя P-1 первой ступени сепарации на орошение низкотемпературного абсорбера также постоянна и принята равной $15,6 \mathrm{~m}^{3} / 4$.

Область оптимальной работы технологической нитки установки определялась по результатам изучения влияния каждого из входных параметров потока пластового газа на составы газа сепарации и нестабильного конденсата в процессе НТА в вариантах: без изменения технологической схемы и установки дополнительного оборудования и с минимальной ее модернизацией включением насоса подачи абсорбента с разделительной емкости первой ступени сепарации.

\section{Результаты расчетов}

Действующим регламентом в настоящее время установлен режим работы установки НТА: расход газа 225 тыс. м $/$, давление 3,75 МПа, температура $30{ }^{\circ} \mathrm{C}$, расход абсорбента $15,6 \mathrm{~m}^{3} /$ ч (при плотности НК $640 \ldots 660$ кг $/ \mathrm{M}^{3}$ массовый расход составляет $10,0 \ldots 10,3$ т/ч, а удельный расход - 69,3 л/тыс. м газа).

Зависимость эффективности сепарации газа и конденсата от термобарических условий процесса НТА

На рис. 2-4 представлены результаты расчётов влияния давления и температуры процесса HТА на удельный выход нестабильного конденсата, удельное извлечение фракции $\mathrm{C}_{3+}$ из газа в НК, массовую концентрацию компонентов $\mathrm{C}_{1}-\mathrm{C}_{2}$ в НК на выходе из абсорбера. Расчеты выполнены для расхода газа 225 тыс. $\mathrm{m}^{3} /$ ч при подаче абсорбента $15,6 \mathrm{~m}^{3} /$ ч.

Как видно из рис. 2, увеличение удельного выхода конденсата происходит как с ростом давления в НТА, так и при снижении температуры в аппарате, но при этом зависимость извлечения компонентов фракции $\mathrm{C}_{3+}$ в нестабильный конденсат от давления характеризуется локальным максимумом при давлении 5,0 ...5,5 МПа с увеличением выхода этой фракции в НК по мере снижения температуры (рис. 3).

Содержание лёгких компонентов $\mathrm{C}_{1}-\mathrm{C}_{2}$ в нестабильном конденсате на выходе из низкотемпературного абсорбера монотонно увеличивается как с ростом давления процесса, так и при снижении температуры входного потока газа (рис. 4).

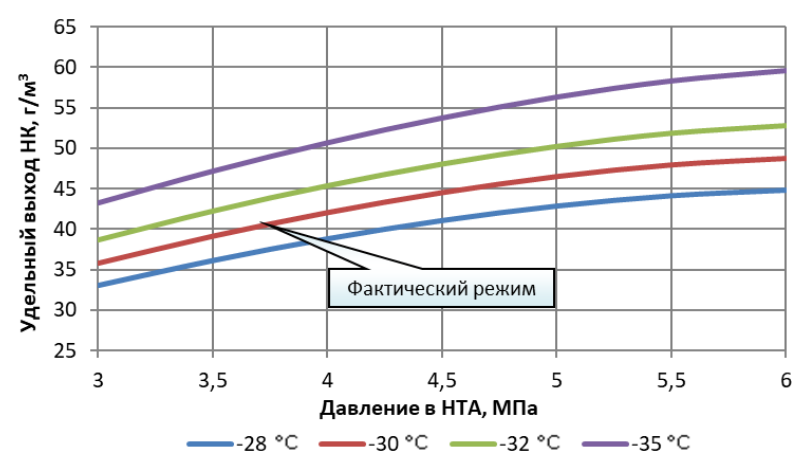

Pис. 2. Зависимость удельного выхода нестабильного конденсата от давления и температуры в низкотемпературном абсорбере

Fig. 2. Dependence of the specific yield of unstable condensate $(U C)$ on pressure and temperature in lowtemperature absorber

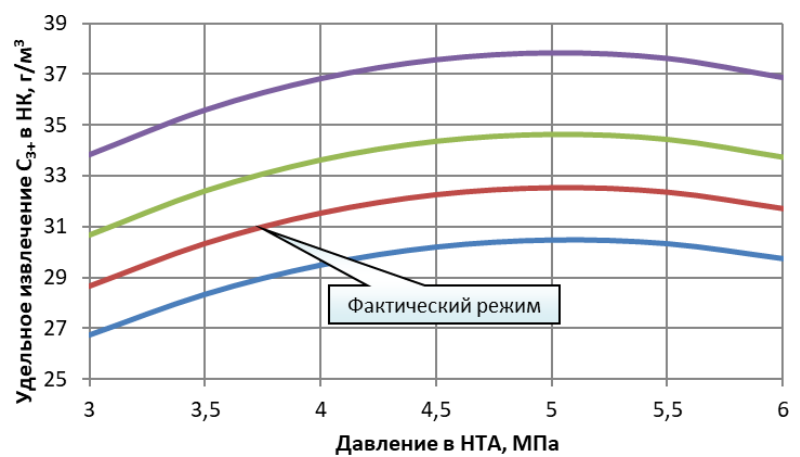

$-28^{\circ} \mathrm{C}-30^{\circ} \mathrm{C}-32^{\circ} \mathrm{C}-35^{\circ} \mathrm{C}$

Pис. 3. Зависимость извлечения компонентов группь $\mathrm{C}_{3+}$ в нестабильный конденсат от давления $u$ температуры в низкотемпературном абсорбере

Fig. 3. Dependence of components $C_{3+}$ extraction in unstable condensate on pressure and temperature in lowtemperature absorber

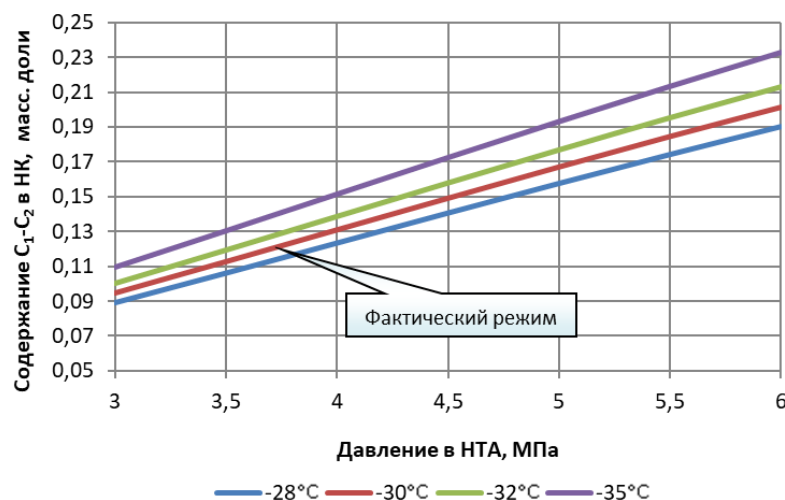

Pис. 4. Зависимость содержания компонентов группь $C_{1}-C_{2}$ в нестабильном конденсате от давления $и$ температуры в низкотемпературном абсорбере

Fig. 4. Dependence of $C_{1}-C_{2}$ components content in unstable condensate on pressure and temperature in lowtemperature absorber

Графики зависимостей на рис. 2-4 показывают, что увеличение выхода НК при давлении процесса выше 5 МПа происходит в основном за счет роста содержания в НК углеводородов $\mathrm{C}_{1}-\mathrm{C}_{2}$, тогда как степень извлечения целевой фракции $\mathrm{C}_{3+}$ снижается. 
С другой стороны, эффективность процесса НТА характеризуется также содержанием фракций $\mathrm{C}_{3+}$ и $\mathrm{C}_{5+}$ в товарном газе. На рис. 5, 6 представлены зависимости содержания фракций $\mathrm{C}_{3+}$ и $\mathrm{C}_{5+}$ в газе сепарации после низкотемпературного абсорбера от термобарических условий в аппарате. Сопоставление зависимостей рис. 3, 6 показывает, что рост извлечения фракции $\mathrm{C}_{3+}$ в нестабильный конденсат при увеличении давления до 5,0 МПа в аппарате происходит за счёт компонентов $\mathrm{C}_{3}-\mathrm{C}_{4}$.

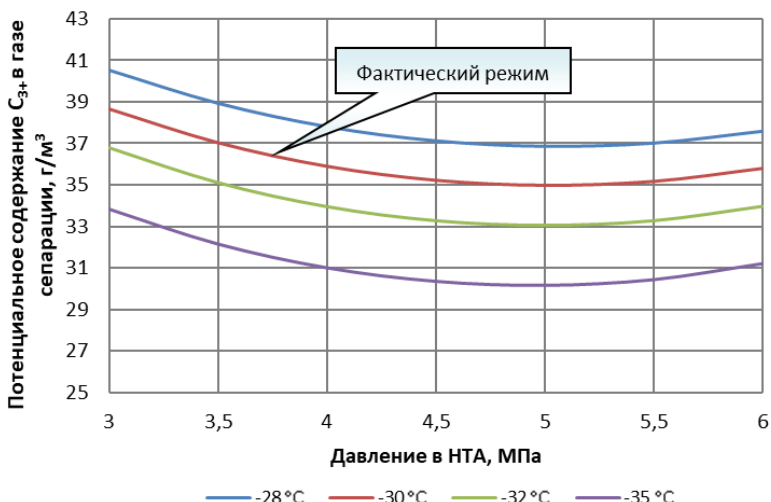

Pис. 5. Зависимость содержания компонентов группь $\mathrm{C}_{3+}$ в газе сепарации от давления и температуры в низкотемпературном абсорбере

Fig. 5. Dependence of components $C_{3+}$ content in separation gas on pressure and temperature in lowtemperature absorber

Вместе с тем следует иметь в виду, что увеличение давления процесса НТА с 4,0 до 5,0 МПа приводит к незначительному повышению уноса с газом сепарации фракции $\mathrm{C}_{5_{+}}$примерно на $0,2 \mathrm{\Gamma} / \mathrm{M}^{3}$ независимо от температуры в абсорбере А-2 (рис. 6) вследствие некоторого «облегчения» компонентного состава НК, подаваемого на орошение из разделительной емкости первой ступени P-1 (табл. 2). Поэтому режимы работы низкотемпературного абсорбера с давлением выше 5 МПа существенно снижают технологическую эффективность процесса НТА и не могут быть рекомендованы для практического применения.

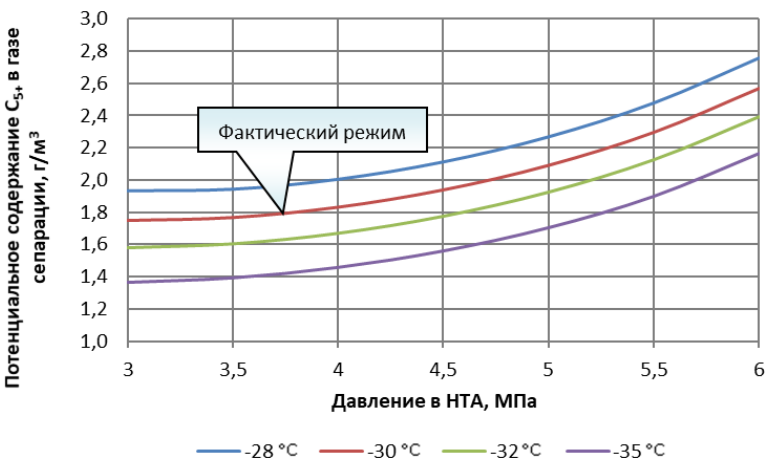

Pис. 6. Зависимость содержания компонентов группь $C_{5+}$ в газе сепарачии от давления и температуры в низкотемпературном абсорбере

Fig. 6. Dependence of components $C_{5+}$ content in separation gas on pressure and temperature in lowtemperature absorber
Таким образом, оптимальные параметры процесса лежат в интервале значений давления 4,5...5,0 МПа и температуры минус $30 \ldots$ минус $35{ }^{\circ} \mathrm{C}$. Температурный режим работы низкотемпературного абсорбера из-за помутнения конденсата при парафинообразовании технологически ограничен минимально допустимым значением температуры - минус $36^{\circ} \mathrm{C}$.

Зависимость эффективности сепарации газа и конденсата в процессе НТА от расхода газа

При исследовании влияния расхода газа расчеты выполнялись при давлении в аппарате 3,75 МПа, температуре минус $30{ }^{\circ} \mathrm{C}$ и подаче абсорбента $15,6 \mathrm{~m}^{3} /$ ч.

Расчеты показали, что при увеличении расхода газа с 200

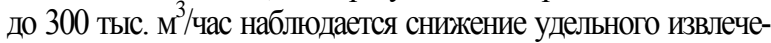
ния целевой фракции $\mathrm{C}_{3+}$ на 4,6 \% с уменышением удельного выхода НК на 11,8 \%. При этом содержание лёгких «газовых» компонентов в НК с увеличением расхода газа питания в заданном диапазоне возрастает на 5,1 \%. Эти закономерности объясняются увеличением скорости газа в аппарате и сокращением времени массообменных процессов в абсорбционной секции. Таким образом, увеличение расхода газа питания ведет к снижению эффективности процесса НТА.

Влияние расхода нестабильного конденсата орошения на эфффективность сепарации газа и конденсата в процессе НTA

Влияние расхода абсорбента - нестабильного конденсата, подаваемого на орошение в абсорбер А-2, на процесс низкотемпературной абсорбции изучалось также при расходе газа 225 тыс. $\mathrm{m}^{3 / 4}$, давлении 3,75 МПа и температуре минус $30{ }^{\circ} \mathrm{C}$ в низкотемпературном абсорбере. В результате расчетов получены зависимости, представленные на рис. 7, из которых следует, что увеличивая орошение, можно добиться повышения выхода НК и удельного извлечения фракции $\mathrm{C}_{3+}$ в НК при снижении содержания газовых компонентов в нестабильном конденсате.

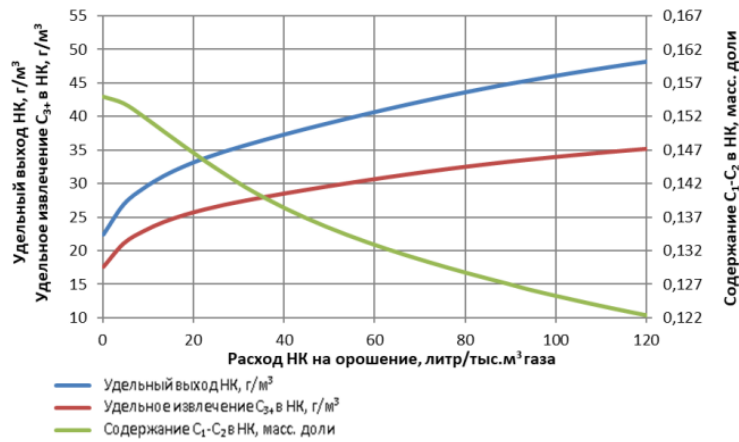

Pис. 7. Зависимости удельного выхода нестабильного конденсата, извлечения компонентов группы $C_{3+}$ в нестабильный конденсат и содержания компонентов $C_{1}-C_{2}$ в нестабильном конденсате от удельного расхода НК на орочение низкотемпературного абсорбера

Fig. 7. Dependences of the specific yield of unstable condensate, the $C_{3+}$ components extraction into unstable condensate and the $C_{1}-C_{2}$ components content in unstable condensate on the specific consumption of unstable condensate for irrigation of lowtemperature absorber 
Важно отметить, что зависимости выхода НК и содержания фракции $\mathrm{C}_{3+}$ в НК при расходе абсорбента свыше 40 л/тыс. ${ }^{3}$ имеют характер монотонного роста, но максимальный расход абсорбента технологически ограничен ресурсом НК, выделяющегося на первой ступени сепарации - подачей 25 т/ч при максимальной производительности одной технологической нитки до 400 тыс. мч и начальном потенциальном содержании конденсата, поэтому при текущей производительности одной технологической нитки по газу 225 тыс. м³/ч обеспечиваемый удельный расход абсорбента не превышает $70 \ldots 75$ л/тыс. м ${ }^{3}$ газа. Дальнейшее увеличение удельного расхода НК потребует привлечения его ресурсов из дополнительных источников, что усложнит технологию процесса и управление им. Поэтому возможности повышения выхода фракций $\mathrm{C}_{3+}$ и $\mathrm{C}_{5+}$ регулированием расхода абсорбента в данном случае весьма ограничены.

Зависимость эфффективности сепарации газа и конденсата в процессе НТА от режима работы разделителя Р-1

Как следует из табл. 2, компонентный состав НК, подаваемого на орошение в абсорбер А-2, сильно зависит от давления в разделителе Р-1. Для оценки влияния давления в разделителе первой ступени Р-1 на удельный выход нестабильного конденсата и удельное извлечение компонентов группы $\mathrm{C}_{3+}$ в процессе НТА были выполнены расчеты сепарации газа питания (после первой ступени) для двух режимов: фактического режима работы низкотемпературного абсорбера (давление 3,75 МПа, температура минус $30{ }^{\circ} \mathrm{C}$ ) и оптимального режима его работы (давление в HТА 5 МПа, температура минус $35^{\circ} \mathrm{C}$ ) при постоянных значениях расхода газа 225 тыс. м $3 / 4$ и подачи нестабильного конденсата на орошение 15,6 $\mathrm{m}^{3} / \mathrm{ч}$. Результаты представлены на рис. 8, 9 .

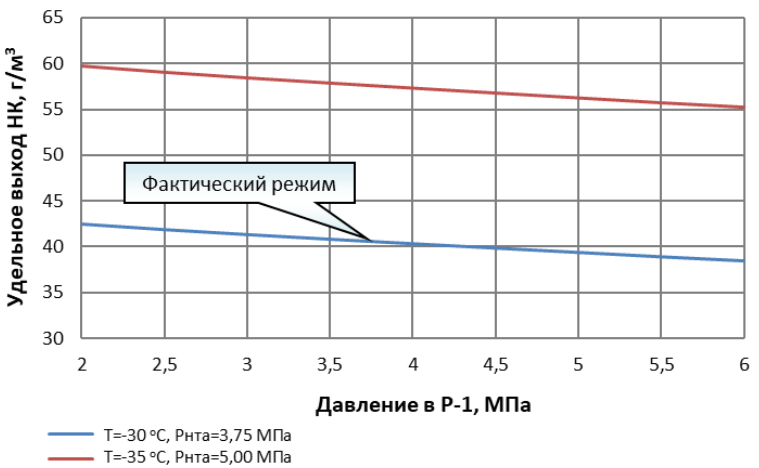

Рис. 8. Зависимость удельного выхода нестабильного конденсата от состава абсорбента, соответствующего давлению в разделителе $P-1$, для фактического и оптимального режимов работы абсорбера $A-2$

Fig. 8. Dependence of the specific yield of unstable condensate on the absorbent composition corresponding to the pressure in the separator $P-1$ for actual and optimal operating modes of the absorber A-2

Как видно из рис. 8, 9, наибольший удельный выход конденсата и наибольшее извлечение фракции $\mathrm{C}_{3+}$ достигается при компонентном составе абсорбен- та - НК, соответствующем давлению в разделителе P-1 2 МПа (табл. 2). Для фактического режима работы абсорбера А-2 применение состава НК орошения, соответствующего давлению в разделителе Р-1 2,0 МПа, по сравнению с НК, состав которого соответствует давлению в разделителе Р-1 3,75 МПа, увеличивает выход НК из абсорбера на 2,04 г/ $\mathrm{m}^{3} \mathrm{c} 40,41$ до 42,45 г/м ${ }^{3}$ (нижняя линия, рис. 8). Для режима работы абсорбера А-2 с абсорбентом, состав которого соответствует давлению в разделителе Р-1 5 МПа, такое увеличение выхода НК составляет уже $3,53 \mathrm{r} / \mathrm{m}^{3}$

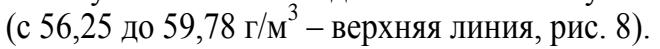

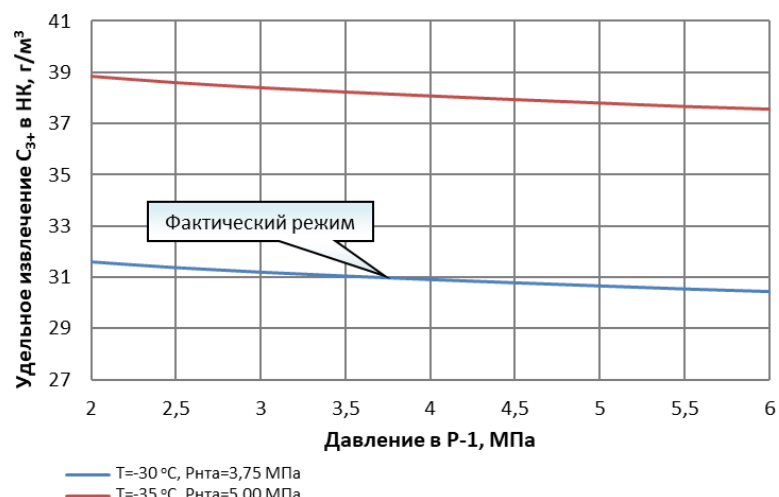

Pис. 9. Зависимость извлечения фракичии $C_{3+}$ в нестабильный конденсат от состава абсорбента, соответствуюшего давлению в разделители $P-1$, для фактического и оптимального режимов работы абсорбера $A-2$

Fig. 9. Dependence of $\mathrm{C}_{3+}$ fraction extraction into unstable condensate on the absorbent composition corresponding to the pressure in the separators $P-1$ for actual and optimal operating modes of the absorber A-2

Аналогично смена состава абсорбента - нестабильного конденсата на орошение, соответствующего давлению в разделителе Р-1 3,75 МПа, на состав НК при 2,0 МПа увеличивает извлечение фракции $\mathrm{C}_{3+}$ в

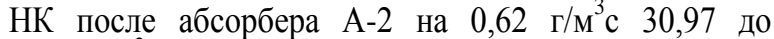
31,59 г/м $\mathrm{M}^{3}$ при фактических режимных параметрах работы абсорбера А-2 (нижняя линия, рис. 9) и на

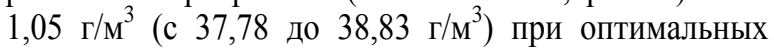
(верхняя линия, рис. 9).

Таким образом, перевод работы установки НТА на оптимальный режим работы (давление и температура в абсорбере А-2 5,0 МПа и минус $35^{\circ} \mathrm{C}$ соответственно) с подачей на орошение НК компонентного состава, соответствующего давлению в разделителе Р-1 2,0 МПа, позволяет поднять выход НК после абсор-

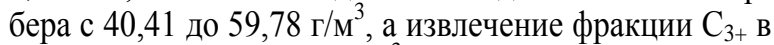
НК - с 30,97 до 38,83 г/M ${ }^{3}$ и соответственно уменьшить унос компонентов $\mathrm{C}_{3+}$ с товарным газом, а также содержание компонентов $\mathrm{C}_{1}-\mathrm{C}_{2}$ в НК.

Однако для закачки НК при давлении 2,0 МПа с разделителя Р-1 в абсорбер А-2 с давлением 5,0 МПа необходима минимальная модернизация установки HТА путем врезки насоса с необходимыми характеристиками по производительности (не менее 15,6 м³/ч) и напору в технологический трубопровод подачи не- 
стабильного конденсата после разделителя Р-1. Она позволит на режиме работы последней ступени сепарации при давлении 5,0 МПа и температуре минус $35{ }^{\circ} \mathrm{C}$ повысить извлечение фракции $\mathrm{C}_{3+}$ в товарный НК с $30,94(30,97)$ до $38,83 \Gamma^{2} \mathrm{~m}^{3}$, т. е. на $25,5 \%$. При этом следует отметить, что изменение компонентного состава абсорбента - НК орошения, слабо повлияло на извлечение фракции $\mathrm{C}_{5+}$ в товарный НК и ее унос с товарным газом: содержание этой фракции в товарном газе остаётся на уровне 1,79 г/м м $^{3}$ для фактического режима эксплуатации и 1,64 г/м $\mathrm{M}^{3}$ для рекомендуемого режима работы НТА. Таким образом, рост извлечения фракции $\mathrm{C}_{3+}$ в товарный НК при смене режима работы абсорбера и компонентного состава абсорбента связан с увеличением доли компонентов $\mathrm{C}_{3}-\mathrm{C}_{4}$ в ее составе.

Сравнение эффрективности сепарации газа на последней ступени в процессах НTA и НTC

Практический интерес представляет оценка эффективности процесса НТА в сравнении с процессом НТС на последней ступени подготовки газа исследуемого состава. Расчеты процесса НТА и НТС выполнялись, как и в предыдущих случаях, при часовом расходе газа 225 тыс. м вался при удельном расходе абсорбента состава, соответствующего регламентному режиму работы установки, - 69,3 л/тыс. м ${ }^{3}$ газа. Результаты расчетов удельных выходов нестабильного конденсата в зависимости от термобарических условий процессов НТА и НТС представлены на рис. 10, из которых следует, что при одинаковых для обоих процессов давлении 3,75 МПа и температуре минус $30{ }^{\circ} \mathrm{C}$ выход НК при использовании в качестве последней ступени НТА

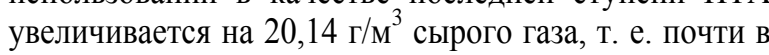
2 раза по сравнению с использованием на последней ступени НТС.

При этом в процессе НТС унос с газом сепарации фракций $\mathrm{C}_{5+}$ и $\mathrm{C}_{3+}$ при давлении в сепараторе $3,75 \mathrm{MПа}$ и температуре минус $30{ }^{\circ} \mathrm{C}$ остаётся на уровне 4,20 и

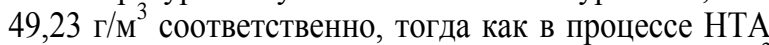
в тех же термобарических условиях - 1,79 и 36,82 г/ $\mathrm{m}^{3}$. Степень извлечения фракций $\mathrm{C}_{5+}$ и $\mathrm{C}_{3+}$ на ступени НТС в товарный НК при этих термобарических условиях составляет 76,3 и 27,9\% соответственно, а на ступени НТА - 87,8 и 46,1 \% соответственно; при давлении 5 МПа и температуре минус $35{ }^{\circ} \mathrm{C}$ унос фракций $\mathrm{C}_{5+}$ и $\mathrm{C}_{3+}$ с газом сепарации несколько сни-

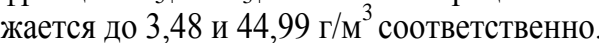

При этих же условиях (5 МПа и минус $35^{\circ} \mathrm{C}$ ) в процессе НТА остаточное содержание фракций $\mathrm{C}_{5+}$ и $\mathrm{C}_{3+}$ в газе сепарации составляет 1,70 и 30,17 г/M $\mathrm{M}^{3}$ соответственно. Эти результаты подтверждают несомненную эффективность применения на последней ступени сепарации процесса НТА.

В целом результаты расчетов процесса НТА на модели УКПГ месторождения Крайнего Севера с технологией НТА позволили выявить те параметры управления процессом сепарации последней ступени, к которым выходные показатели (извлечение целевой фракции $\mathrm{C}_{3+}$ в $\mathrm{HK}$ и содержание компонентов $\mathrm{C}_{1}-\mathrm{C}_{2}$ в
НК) в конкретных технологических условиях наиболее чувствительны, - это температура, давление в аппарате и состав подаваемого абсорбента.

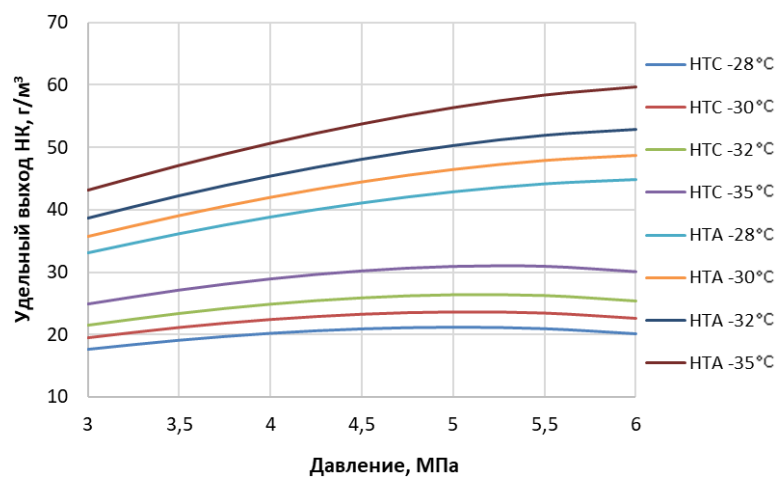

Рис. 10. Зависимости удельного выхода НК от давления и температуры в процессах НTA и НTC

Fig. 10. Dependences of UC specific yield on pressure and temperature in LTA and LTS

Влияние расхода газа на эффективность процесса НТА оказалось относительно слабым, несмотря на широкий диапазон его изменения. Кроме того, на практике возможности регулирования расхода газа питания низкотемпературного абсорбера (газа сепарации первой ступени) в промысловых условиях ограничены текущим планом добычи. Удельный расход абсорбента принимается близким к максимально возможному и ограничен содержанием конденсата в сыром газе и ресурсом НК, выделяемым на первой ступени сепарации. Следовательно, расход газа питания и удельный расход абсорбента (подача НК орошения) не могут рассматриваться в полной мере как инструмент регулирования эффективности процесса НТА.

\section{Заключение}

Для решения технологической задачи повышения удельного содержания фракции $\mathrm{C}_{3+}$ в товарном нестабильном конденсате после последней ступени промысловой установки подготовки конденсатсодержащего газа смоделированы процессы НТА и НТС. Показано, что использование процесса НТА на последней ступени сепарации для рассматриваемого состава сырого газа обеспечивает выход целевой фракции примерно в 2 раза больше в широком диапазоне давлений и температур по сравнению с процессом НТС. При часовом расходе сырого газа 225 тыс. м/час оптимизацией термобарических параметров процесса НТА - изменением давления с 3,75 до 5,0 МПа и температуры с минус 30 до минус $35{ }^{\circ} \mathrm{C}-$ извлечение фракции $\mathrm{C}_{3+}$ в нестабильный товарный конденсат повышается на $6,6 r / \mathrm{m}^{3}$ сырого газа без модернизации технологической схемы установки, или на 35 т/сутки, с соответствующим сокращением уноса этой фракции с товарным газом.

При таком же изменении режима работы низкотемпературного абсорбера, но с «утяжелением» компонентного состава абсорбента - НК, получаемого в разделителе первой ступени P-1 при давлении 2,0 МПа, извлечение фракции $\mathrm{C}_{3+}$ в нестабильный 


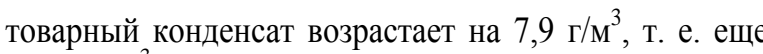
на 1,3 г/м ${ }^{3}$, или 7 т/сутки дополнительно. Но реализация такого режима уже требует минимальной модернизации установки НТА путем врезки насоса в техно-

\section{СПИСОК ЛИТЕРАТУРЫ}

1. Campbell J.M. Gas conditioning and processing. V. 2: The equipment modules. $7^{\text {th }}$ ed. - USA: Campbell Petroleum Series, 1992. $444 \mathrm{p}$.

2. Mokhatab S., Poe W.A., Mak J.Y. Handbook of natural gas transmission and processing. Principles and Practices. $3^{\text {rd }}$ ed. - Waltham: Gulf Professional Publishing. Elsevier, 2015. - 597 p.

3. Modeling of liquid separators working as and gas condensate preparation unit in low-temperature separation technology / I. Dolganov, M. Pisarev, E. Ivashkina, I. Dolganova // Petroleum and Coal. - 2015 - V. 57. - P. 328-335.

4. Берлин М.А., Гореченков В.Г., Капралов В.П. Квалифицированная первичная переработка нефтяных и природных углеводородных газов. - Краснодар: Советская Кубань, 2012. $265 \mathrm{c}$.

5. Истомин В.А. Низкотемпературные процессы промысловой обработки природных газов. Ч. 2. - М.: ИРЦ Газпром, 1999. $58 \mathrm{c}$.

6. Кубанов А.Н. Интенсификация промысловой низкотемпературной обработки природных газов на северных месторождениях: дис. .... канд. тех. наук. - М., 1998. - 111 с.

7. Арнольд К., Стюарт М. Справочник по оборудованию для комплексной подготовки газа. Промысловая подготовка углеводородов / пер. с англ. - М.: ООО «Премиум Инжиниринг», 2009. $-630 \mathrm{c}$.

8. Особенности технологических процессов промысловой подготовки природного газа с низким конденсатным фактором А.В. Дунаев, В.А. Истомин, А.Н. Кубанов, В.В. Моисеев, Д.М. Федулов // Газовая промышленность. - 2015. - № 11. C. 80-83.

9. Современное состояние технологий промысловой подготовки газа газоконденсатных месторождений / А.В. Прокопов, А.Н. Кубанов, В.А. Истомин, Д.М. Федулов, Т.С. Цацулина // Вести газовой науки: Актуальные проблемы добычи газа. 2015. - № 3. - C. 100-108.

10. Kidnay A.J., Parrish W.R. Fundamentals of natural gas processing. - Boca Raton: CRC Press, 2006. - 464 p.

11. Николаев О.А., Кабанов О.П., Цветков Н.А. Степень извлечения и остаточное содержание углеводородов $\mathrm{C}_{5+}$ в газе сепарации газоконденсатных месторождений // НефтеГазоХимия. -2016 . - № 2. - С. 64-70.

12. Optimization of the thermobaric conditions of the gas treatment by low-temperature separation / D. Mukhametova, V. Kolchin, V. Kurochkin, R. Ismagilov // Petroleum engineering «Oil and gas fields development». -2018 . - V. 4. - P. 79-88.

13. Специфика промысловой подготовки газов ачимовских залежей / А.В. Прокопов, А.Н. Кубанов, В.А. Истомин, логический трубопровод подачи нестабильного конденсата орошения с разделителя Р-1 в низкотемпературный абсорбер, которая экспертно вполне оправдана и окупаема в короткие сроки.

Д.Н. Снежко, А.Н. Чепурнов, А.К. Акопян // Вести газовой науки: Актуальные проблемы добычи газа. - 2018. - № 1. C. 226-234

14. Кубанов А.Н., Туревский Е.Н., Шевелев С.А. Опыт эксплуатации технологии ПНТА и перспективы внедрения новых способов извлечения жидких углеводородов // Оценка эффективности научно-технических решений, реализованных на нефтегазодобывающих объектах ОАО «Газпром»: материалы науч.-тех. совета ОАО «Газпром». - М.: ИРЦ Газпром, 1999. C. $134-143$

15. Прокопов А.В., Истомин В.А. Абсорбционные технологии промысловой подготовки газоконденсатных газов // Вести газовой науки: Актуальные проблемы добычи газа. - 2016. № 2. - С. 165-173.

16. Прокопов А.В. Совершенствование технологии промысловой подготовки газа газоконденсатных месторождений с высоким конденсатным фактором: дис. ... канд. техн. наук. - М., 2019. - $120 \mathrm{c}$.

17. Прокопов А.В., Истомин В.А., Федулов Д.М. Разработка новой технологии низкотемпературной абсорбции для подготовки природного газа газоконденсатных месторождений // Химическая промышленность сегодня. - 2017. - № 6. - С. 37-47.

18. Wang J., Smith R. Synthesis and optimization of low-temperature gas separation processes // Industrial and engineering chemistry research. -2005 . - V. 44. - P. 2856-2870.

19. Прокопов А.В., Истомин В.А., Федулов Д.М. Выделение углеводородов $\mathrm{C}_{3+}$ из газоконденсатной смеси при промысловой подготовке пластового флюида // Вести газовой науки: Актуальные проблемы добычи газа. - 2016. - № 4 (28). C. 202-206

20. Modes of gas and gas condensate preparation unit in lowtemperature separation technology modeling / I. Dolganov, M. Pisarev, E. Ivashkina, I. Dolganova // Petroleum and Coal. 2014. - V. 56. - P. 182-187.

21. Maddox J.R.N., Bretz K.E. Turbo-expander applications in natural gas processing // Journal of Petroleum Technology. - 1976. V. 5. - P. 611-613.

22. Методический подход для расчётного исследования процессов промысловой низкотемпературной сепарации с турбодетандерами / М.А. Воронцов, Д.М. Федулов, А.С. Грачев, А.В. Прокопов, В.Ю. Глазунов // Вести газовой науки: Актуальные проблемы добычи газа. - 2016. - № 2 (26). - С. 105-111.

23. Mathematical simulation flow-temperature gas separation A. Bunaev, I.M. Dolganov, I.O. Dolganova, A. Vladescu // Petroleum and Coal. - 2017. - V. 59. - P. 210-219.

Поступила 22.10.2020 г.

\section{Информация об авторах}

Кутуков В.B., студент-магистрант кафедры разработки и эксплуатации газовых и нефтегазоконденсатных месторождений Уфимского государственного нефтяного технического университета.

Пономарёв А.И., доктор технических наук, профессор, заведующий кафедрой разработки и эксплуатации газовых и нефтегазоконденсатных месторождений Уфимского государственного нефтяного технического университета.

Чеботарёв В.В., кандидат технических наук, профессор кафедры разработки и эксплуатации газовых и нефтегазоконденсатных месторождений Уфимского государственного нефтяного технического университета. 
UDK 622.279.8:66.081.2

\title{
EVALUATION OF TEMPERATURE AND PRESSURE CONDITIONS EFFECT ON LOW-TEMPERATURE ABSORPTION EFFECTIVENESS AT GAS TREATMENT ON THE FAR NORTH FIELD
}

\author{
Vladislav V. Kutukov', \\ Kutukov_Vlad@rambler.ru \\ Alexander I. Ponomarev', \\ pnmrv@mail.ru \\ Viktor V. Chebotarev1, \\ kafedrargkm@mail.ru \\ 1 Ufa State Petroleum Technical University, \\ 1, Kosmonavtov street, Ufa, 450062, Russia.
}

Relevance. Gas condensate is a valuable raw material for oil refining and petrochemical production, therefore an increase in the degree of its extraction from gas of the gas condensate fields at field installations is an important scientific and technical problem.

The aim of the study is to substantiate the possibility of increasing the yield of unstable condensate $-C_{3+}$ fraction from the gas stream at operating complex gas and condensate treatment plant of one of the oil and gas condensate fields in the Far North by changing the operating parameters of the low-temperature absorption.

The object: field plant of low-temperature absorption.

Method: simulations of separation and low-temperature absorption in the software package «PetroSim».

Results. The authors have studied the influence of pressure and temperature, gas flow, specific flow rate and composition of the absorbent - unstable condensate, on the efficiency of the gas and condensate preparation at complex gas and condensate treatment plant computer model of low-temperature absorption and low-temperature separation. It is shown that the low-temperature absorption application at the last stage of separation for the considered raw gas composition provides an output of the target $C_{3+}$ fraction approximately two times greater in a wide range of pressures and temperatures compared to the low-temperature separation. The possibility of increasing the degree of the $\mathrm{C}_{3+}$ fraction extraction into commercial unstable condensate at the last stage of separation in the low-temperature absorption by $21 \%$ is justified only by optimizing the operating parameters of the unit without changing the technological scheme. At the raw gas flow rate of $225 \mathrm{~m}^{3} /$ hour the $C_{3+}$ fraction extraction into unstable commercial condensate increases to $6,6 \mathrm{~g} / \mathrm{m}^{3}$ of raw gas without modernization of the technological scheme of the unit, and this is an additional over $35 \mathrm{MT} /$ day $\mathrm{C}_{3+}$ fraction with a corresponding reduction in its entrainment with the product gas, by optimization of low-temperature absorption thermobaric parameters (change of pressure from 3,75 to $5,0 \mathrm{MPa}$ and temperature from minus 30 to minus $35^{\circ} \mathrm{C}$ ). When "weighting» the component composition of the absorbent by reducing the pressure in the unstable condensate separator of the first stage separation the degree of extraction of $\mathrm{C}_{3+}$ fraction in the product unstable condensate in the low-temperature absorber increases by $25 \%$, i. e. $6,6 \mathrm{~g} / \mathrm{m}^{3}$, and this is $7 \mathrm{MT} /$ day in addition with a corresponding further reduction of its content in the product gas. However, the implementation of such a regime already requires minimal modernization of the low-temperature absorption unit by inserting a pump into the process pipeline for supplying unstable irrigation condensate from the $R-1$ separator to a low-temperature absorber, which is repaid in a short time.

\section{Key words:}

Gas and condensate field, gas field treatment, low-temperature absorption, computer simulation,

temperature and pressure conditions, separation gas, unstable condensate output, selective absorbent, pump.

\section{REFERENCES}

1. Campbell J.M. Gas conditioning and processing. Vol. 2: The equipment modules. $7^{\text {th }}$ ed. USA, Campbell Petroleum Series, 1992 $444 \mathrm{p}$.

2. Mokhatab S., Poe W.A., Mak J.Y. Handbook of natural gas transmission and processing. Principles and Practices. $3^{\text {rd }}$ ed. Waltham, Gulf Professional Publ. Elsevier, 2015. 597 p.

3. Dolganov I., Pisarev M., Ivashkina E., Dolganova I. Modeling of liquid separators working as and gas condensate preparation unit in low-temperature separation technology. Petroleum and Coal, 2015, vol. 57, pp. 328-335.

4. Berlin M.A., Porechenkov V.G., Kapralov V.P. Kvalifitsirovannaya pervichnaya pererabotka neftyanykh $i$ prirodnykh uglevodorodnykh gazov [Qualified primary processing of oil and natural hydrocarbon gases]. Krasnodar, Soviet Kuban Publ., 2012. 265 p.

5. Istomin V.A. Nizkotemperaturnye protsessy promyslovoy obrabotki prirodnykh gazov. Ch. 2 [Low-temperature processes of field processing of natural gases. P. 2]. Moscow, IRTS Gazprom Publ., 1999. $58 \mathrm{p}$.

6. Kubanov A.N. Intensifikatsyia promyslovoy nizkotemperaturnoy obrabotki prirodnykh gazov na severnykh mestorozhdeniyakh.
Diss. Kand. nauk [Intensification of field low-temperature treatment of natural gases in the Northern fields. Cand. Diss.]. Moscow, 1998. $111 \mathrm{p}$

7. Arnold K., Stiuart M. Spravochnik po oborudovaniyu dlya kompleksnoy podgotovki gaza. Promyslovaya podgotovka uglevodorodov [Handbook of equipment for complex gas treatment. Field preparation of hydrocarbons]. Moscow, Premium Inzhiniring Publ., 2009. $630 \mathrm{p}$.

8. Dunaev A.V., Istomin V.A., Kubanov A.N., Moiseev V.V., Fedulov D.M. Features of technological processes of field preparation of natural gas with a low condensate factor. GAS industry of Russia, 2015, no. 3, pp. 80-83. In Rus.

9. Prokopov A.V., Kubanov A.N., Istomin V.A., Fedulov D.M., Tsatsulina T.S. State-of-art technologies for gas treatment at gascondensate fields. News of gas science, 2015, no. 3, pp. 100-108. In Rus.

10. Kidnay A.J., Parrish W.R. Fundamentals of natural gas processing. Boca Raton, CRC Press, 2006. $464 \mathrm{p}$.

11. Nikolaev O.A., Kabanov O.P., Tsvetkov N.A. Extent of extraction and the residual content of $\mathrm{C} 5+$ hydrocarbons in produced gas of gas-condensate fields. OIL\&GAS CHEMISTRY, 2016, no. 2, pp. 64-70. In Rus. 
12. Mukhametova D., Kolchin V., Kurochkin V., Ismagilov R. Optimization of the thermobaric conditions of the gas treatment by low-temperature separation. Petroleum engineering «Oil and gas fields development», 2018, vol. 4, pp. 79-88.

13. Prokopov A.V., Kubanov A.N., Istomin V.A., Snezhko D.N., Chepurnov A.N., Akopian A.K. Specifics of field gas preparation in Achimov deposits. News of gas science, 2018, no. 1, pp. 226-234. In Rus.

14. Kubanov A.N., Turevsky E.N., Shevelev S.A. Opyt ekspluatatsii tekhnologii PNTA i perspektivy vnedreniya novykh sposobov izvlecheniya zhidkikh uglevodorodov [Operating experience of FLTA technology and possibility for introducing new methods for extracting liquid hydrocarbons]. Otsenka effektivnosti nauchnotekhnicheskikh resheniy, realizovannykh na neftegazodobyvayushchikh obektakh OAO «Gazprom». Materialy nauchnotekhnicheskogo soveta $O A O$ «Gazprom» [Evaluating the effectiveness of scientific and technical solutions implemented at JSC «Gazprom» oil and gas production facilities. Materials of the scientific and technical council of Open joint stock company «Gazprom»]. Moscow, Information and advertising Center Gazprom, 1999. pp. 134-143.

15. Prokopov A.V., IstominV. A. Absorption techniques for field preparation of wet gases. News of gas science, 2016, no. 2, pp. 165-173. In Rus.

16. Prokopov A.V. Sovershenstvovanie tekhnologii promyslovoy podgotovki gaza gazokondensatnykh mestorozhdeniy s vysokim kondensatnym faktorom. Dis. Kand. nauk [Improving the technology of field treatment of gas from gas condensate fields with a high condensate factor. Cand. Diss.]. Moscow, 2019. 120 p.

17. Prokopov A.V., Istomin V.A., Fedulov D.M. Development of new low-temperature absorption technologies for the treatment of natural gas from gas condensate fields. Chemical industry today, 2017, no. 6, pp. 37-47. In Rus.

18. Wang J., Smith R. Synthesis and optimization of low-temperature gas separation processes. Industrial and engineering chemistry research, 2005, vol. 44, pp. 2856-2870.

19. Prokopov A.V., Istomin V.A., Fedulov D.M. Separation of $\mathrm{C}_{3+}$ hydrocarbons from gas-condensate mixture during field treatment of reservoir fluid. News of gas science, 2016, no. 4, pp. 202-206. In Rus.

20. Dolganov I., Pisarev M., Ivashkina E., Dolganova I. Modes of gas and gas condensate preparation unit in low-temperature separation technology modeling. Petroleum and Coal, 2014, vol. 56, pp. 182-187.

21. Maddox J.R.N., Bretz K.E. Turbo-expander applications in natural gas processing. Journal of Petroleum Technology, 1976, no. 5, pp. 611-613.

22. Vorontsov M.A, Fedulov D.M., Grachev A.S., Prokopov A.V., Glazunov V.Yu. Methodical approach for the calculated research processes of field low-temperature separation with turboexpanders. News of gas science, 2016, no. 2, pp. 105-111. In Rus.

23. Bunaev A., Dolganov I. M., Dolganova I. O., Vladescu A. Mathematical simulation of low-temperature gas separation. Petroleum and Coal, 2017, vol. 59, pp. 210-219.

Received: 22 October 2020.

\section{Information about the authors}

Vladislav V. Kutukov, master student, Ufa State Petroleum Technical University.

Alexander I. Ponomarev, Dr. Sc., professor, Ufa State Petroleum Technical University.

Viktor V. Chebotarev, Cand. Sc., professor, Ufa State Petroleum Technical University. 